Vietnam Journal of Mechanics, VAST, Vol.34, No. 4 (2012), pp. 225 - 236

\title{
ATTENUATION COEFFICIENT FOR SURFACE ACOUSTIC WAVES IN FLUID REGION
}

\author{
Bui Thu Hang, Bui Duc Tung, Nguyen Tien Dat, Chu Duc Trinh \\ University of Engineering and Technology, VNU, Vietnam
}

\begin{abstract}
In recent years, surface acoustic wave devices used in bio-sensing applications have demonstrated high sensitivity in the detection of fluid properties such as density, viscosity, stream velocity. In this paper, a more effective measurement of the SAW sensor structure is presented. It is reported that at density of $6 \mathrm{~g} / \mathrm{cm}^{3}$, the amplitude of mechanical wave is excited while for electrical signal, attenuation at $3 \mathrm{~g} / \mathrm{cm}^{3}$ reaches a peak. In our analysis, single-crystal Aluminium Nitride substrate is used. Several parameters of leaky waves including displacement, decay constant in the liquid media are analyzed.

Keyword: Rayleigh - Surface Acoustic Wave sensor (R-SAW), liquid sensor, Aluminium Nitride.
\end{abstract}

\section{INTRODUCTION}

Saw devices have been applied for sensing chemical and physical features in gas and liquid phases. A mechanical wave in the piezoelectric crystal which is generated by metal electrodes or interdigital transducers (IDTs) placed travels through substrate $[1,2]$. It includes a Rayleigh and a shear mode which propagate through the surface as shown in Fig. 1. The Rayleigh mode, termed Rayleigh wave, is a combination of longitudinal
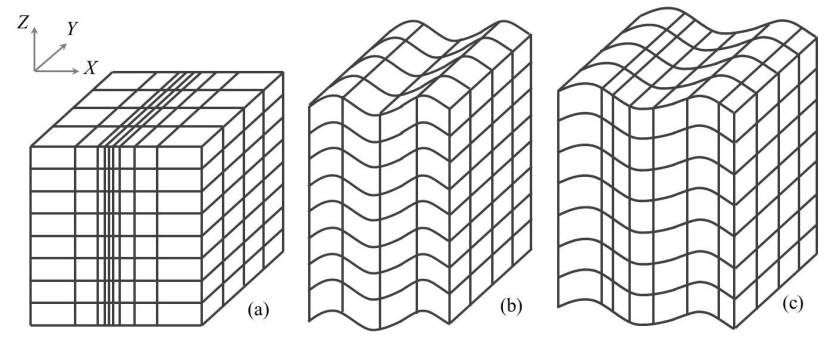

Fig. 1. Acoustic wave propagation direction in Cartesian coordinate system.

(a) Compressional or longitudinal; (b) Shear vertical; (c) Shear horizontal [3].

and shear vertical particle displacement while the shear mode, termed Shear Horizontal Surface Acoustic Wave (SH-SAW), is a shear horizontal wave on the surface [4-6]. 
While the shear component is very attractive for liquid sensors, the presence of the normal displacement component is a reason why Rayleigh-SAW devices are poorly suited for liquid sensing applications [2]. In contact of the solid-liquid medium, the shear vertical, a component of the Rayleigh, and shear horizontal wave couple into the liquid, generate compression waves and lead to dissipated power and extreme attenuation of SAWs. If the contact is too large, these components are able to attenuate excessively and change Rayleigh wave features. On the other hand, surface particles do not move in ellipses in planes being normal to the surface and parallel to the direction of propagation $[7,8]$. Therefore, for liquid sensing applications, the longitudinal component should have inappreciable attenuation. In other words, decay constant along the $X_{3}$-axis is small in the fluid domain. In a previous paper, a new vertical structure was presented to reduce this impact [9]. Its key feature is that the contact area of the solid-liquid which closes to surface is smaller (as shown in Fig. 2).

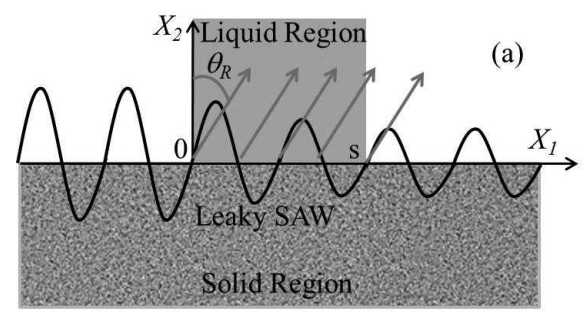

(a) Cross-sectional view of the typical structure

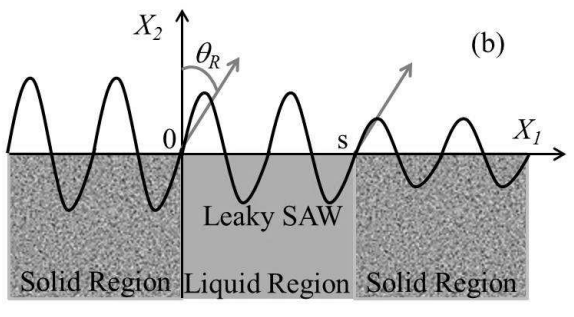

(b) Cross-sectional view of the vertical structure

Fig. 2. SAW radiation into fluid domain in 2D.

This Rayleigh surface acoustic wave structure for characterizing a liquid is sensitive to the different liquids deposited in the well (specifically density changes). Furthermore, as the used Lithium Niobate $\left(\mathrm{LiNbO}_{3}\right)$ substrate exhibited a low frequency response, it was not appropriate for the engineering with micro-electromechanical systems $[10,11]$.

This work proposes a measurement method of SAW devices on single-crystal AlN substrate to detect liquid density range. The fundamental of acoustic wave in the stationary and moving fluid static is analyzed. Also, phase shift and amplitude ratio of mechanical waves and electrical output signal are investigated.

\section{FUNDAMENTALS OF ACOUSTIC WAVE SENSORS FOR LIQUID}

To satisfy the stress-free boundary, compression and shear waves propagate together on the substrate. It is assumed that the generalized surface acoustic wave propagates in the $\left(\widehat{X_{1}}, \widehat{X_{2}}\right)$ direction and has a displacement profile $u_{1}$ which varies with the depth $X_{3}$ of the single-crystal as:

$$
u\left(X_{1}, X_{2}, X_{3}, t\right)=\left[W_{1} \widehat{X_{1}}+W_{2} \widehat{X_{2}}+W_{3} \widehat{X_{3}}\right] e^{j b k x_{3}} e^{i k\left(l_{1} x_{1}+l_{2} x_{2}-\nu t\right)}
$$


where $k$ is the wave number, $\nu$ is the velocity of the wave, $b$ is the decay constant of the wave in the $\widehat{X_{3}}$ direction and $\left(l_{1}, l_{2}\right)$ is the set of propagation direction along the surface. The component $\widehat{X_{3}}$ is perpendicular to the surface and $W_{1}, W_{2}, W_{3}$ represent displacement amplitudes of the $\widehat{X_{1}}, \widehat{X_{2}}$ and $\widehat{X_{3}}$ directions, respectively. It is supposed that there exists a liquid medium positioning in the propagation path with the coordinate system as shown in Fig. 3 .

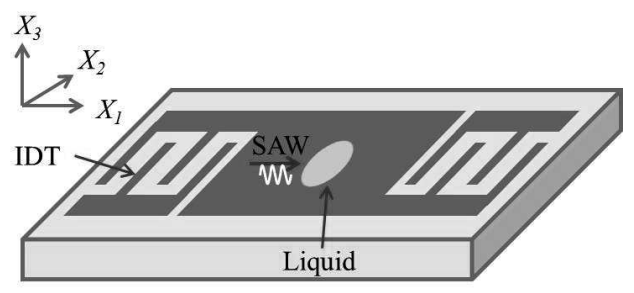

Fig. 3. The coordinate system is used in calculations [6].

The Rayleigh wave is characterized by the lack of transversal component. Thus Eq. 1 omits $\widehat{X_{2}}$ and $l_{1}$ equals to unit. Hence, traveling wave form is independent of the $X_{2}$ coordinate. Since the displacement component $u_{2}$ is removed, $l_{2}$ is zero $[2,12]$.

\subsection{Acoustic wave in the stationary fluid domain}

The traveling wave solutions are sought in the form [6]:

$$
\begin{aligned}
u_{1}^{f} & =W_{1} e^{j b_{f} k x_{3}} e^{j k\left(x_{1}-\nu t\right)} \\
u_{3}^{f} & =W_{3} e^{j b_{f} k x_{3}} e^{j k\left(x_{1}-\nu t\right)} \\
\phi^{f} & =W_{2} e^{j b_{f} k x_{3}} e^{j k\left(x_{1}-\nu t\right)}
\end{aligned}
$$

Here, $b_{f}= \pm \sqrt{\frac{\rho^{f} \nu^{2}-c^{f}}{c^{f}}}$ is the decay constant of the wave in the $\widehat{X}_{3}$ direction and $\alpha=\frac{W_{1}}{W_{3}}=\frac{\rho^{f} \nu^{2}-c^{f} b_{f}^{2}}{c^{f} b_{f}}=\frac{1}{b_{f}}$ is the relation coefficient. $\rho^{f}$ is the fluid density and $c^{f}$ is the elastic constant of the fluid. The fluid viscosity is ignored in the traveling wave solutions.

\subsection{Acoustic wave in the moving fluid domain}

It is assumed that the flow direction coincides with the wave propagation as $\Omega=$ $\beta(t)$. Hence, the displacement component is written:

$$
u_{3}^{f}=W_{3} e^{j b_{f} k x_{3}} e^{j k\left(x_{1}-\nu t\right)}+\beta
$$

The wave propagation in a non-piezoelectric and piezoelectric media is described:

$$
\rho^{f} \frac{\partial^{2} u_{i}}{\partial t^{2}}=\sum_{j, k, l=1}^{3} c_{i j k l} \frac{\partial^{2} u_{k}}{\partial x_{j} \partial x_{l}}
$$

where $\rho$ is the mass density of material and $c_{i j k l}$ are the elastic stiffness tensors (as shown 
in Appendix), respectively. When the flow function is constant or linear, results of the displacement amplitude ratio are similar with the stationary liquid. With the other cases, substituting into Eq. 4, it can be expressed as:

$$
b_{f} W_{1}-W_{3}=\frac{1}{\nu^{2} k^{2}} \dot{\beta}
$$

Consequently, these displacement components in the stationary and moving fluid static depend on density. In the output, the transducer converts vibrations of acoustic waves into electrical signals. Also, the potential is influenced by the mechanical displacement $u_{i}$. Therefore, the amplitude and phase of output are changed by any perturbation of the SAW.

\section{DESIGN PARAMETERS AND MEASUREMENT METHOD OF LIQUID SENSING SYSTEM}

In this section, the R-SAW sensor configuration, the system configuration on singlecrystal AlN substrate and the measurement method are described.

\subsection{Structural parameters}

Based on previous results, when the vertical well reduces the contact area between solid and liquid, it drives to restrict the excessive attenuation phenomenon [9]. As the surface wave amplitude decreases exponentially with increasing distance from the surface, it is possible to avoid the wave reflection at the bottom if wavelength is larger than substrate depth [2]. So, the thickness of the chosen single-crystal Aluminium Nitride (AlN) substrate is longer in the simulation. Additionally, it is able to propagate SAWs more swiftly, exhibit

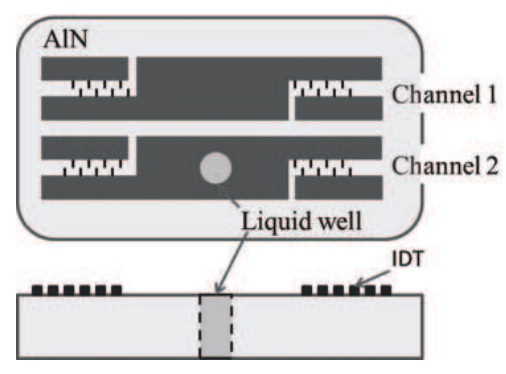

Fig. 4. Schematic illustration of two-channel SAW sensor and liquid well position.

both higher frequency and compatibility in micro-electromechanical systems (MEMS) [5]. For the leaky SAWs generated by piezoelectric materials like Lithium Niobate $\left(\mathrm{LiNbO}_{3}\right)$, Lithium Tantalate $\left(\mathrm{LiTaO}_{3}\right)$ and quartz, $K^{2}$ is larger and the temperature stability for certain orientation is better [13-15]. The liquid sensing system consists of two channels: a reference channel (Channel 1) and a sensing channel (Channel 2).

Fig. 4 shows the top view and the cross-section of the R-SAW sensor. Six IDT pairs for input and output are covered by Aluminium. The well pierces through the center of the Channel 2. Sample liquids are poured onto the well. With the multiple channel structure, 
the sensor can simultaneously measure the changeable electrical and mechanical properties of sample liquids. The density products are obtained from the differential phase shift between the reference channel and the sensing channel and amplitudes of both channels are detected.

Design parameters of two channels are as follows in Tab. 1. Each channel includes two-port and a delay line. The substrate is made of single-crystal AlN in class $6 \mathrm{~mm}$ symmetry. The stiffness, piezoelectric constant and permittivity of the material are shown fully in the appendix. To build a 3D model of single-crystal AlN substrate, a 3D domain of size $400 \mu \mathrm{m}$ along $X$-axis, $200 \mu \mathrm{m}$ along $Y$-axis and thickness along $Z$-axis is created.

Table 1. Design parameters of SAW device

\begin{tabular}{|l|l|r|r|}
\hline Parameters & Symbol & Value & Unit \\
\hline IDT period (acoustic wavelength) & $\Lambda$ & 40 & $\mu \mathrm{m}$ \\
Number of finger pairs & $N$ & 3 & \\
Aperture length & $L$ & 100 & $\mu \mathrm{m}$ \\
AlN thickness & $h$ & 50 & $\mu \mathrm{m}$ \\
Center frequency & $f_{0}$ & 143 & $\mathrm{MHz}$ \\
SAW velocity & $\nu$ & 5720 & $\mathrm{~m} / \mathrm{s}$ \\
Well diameter & $D$ & 30 & $\mu \mathrm{m}$ \\
\hline
\end{tabular}

Liquids are deionized water and water/glycerol mixtures $(\mathrm{W} / \mathrm{G})$. They are classified into two groups: The Standing Group including W/G densities within the range of 1 to 13 $\mathrm{g} / \mathrm{cm}^{3}$, and the Moving Group consisting of deionized water with velocity $\nu=0, \nu_{z}=10$ and $2 t \mu \mathrm{m} / \mathrm{s}$ where $t$ is the time. The piezoelectric surfaces of the developed models are meshed with maximum element size of $32 \mu \mathrm{m}$ and the IDT boundaries are meshed with a maximum element size of $8 \mu \mathrm{m}$. These parameters provide a much denser mesh at the top of the model which is essential to achieve a high accuracy in simulating the SAW propagation. A sinusoidal voltage $10 \mathrm{~V}$ of frequency $143 \mathrm{MHz}$ is applied to the input IDT to generate the needed SAWs. The output voltages in both cases are acquired at the alternating fingers of the output IDT.

\subsection{Measurement method}

The relation between input and output is given by the frequency response function $H$ which is the Fourier transform of the unit impulse response function of the system. It can be calculated optimally from the input power spectrum density $P_{X X}$ and the cross power spectral density $P_{X Y}$ as the following equation [16]:

$$
H(f)=\frac{P_{X Y}(f)}{P_{X X}(f)}
$$

The signal attenuation is measured with the first output IDT. As it is long, a used larger time window is to avoid scattering from substrate edges. It applies the geometry 
scaling factor and performed Turkey windowing for both with and without well signals $[17,18]$.

\section{RESULTS AND DISCUSSIONS}

Simulation time of two R-SAW channels is $1 \mu$ s for the stationary group, $200 \mathrm{nsec}$ for the moving group and a time step is 0.09 nsec.

\subsection{Investigating Stationary Group}

Mode profiles in $Z$ and $X$ coordinate are illustrated in Fig. 5. These modes are referred to as the Rayleigh waves because $Z$ component varies across the thickness of devices and $X$ component which is in the propagation direction involves changes in the substrate thickness. The shear wave going through the fluid domain disperses part of the energy in the longitudinal direction. However, generated compression wave increases inappreciably excepting density equals to $6 \mathrm{~g} / \mathrm{cm}^{3}$.

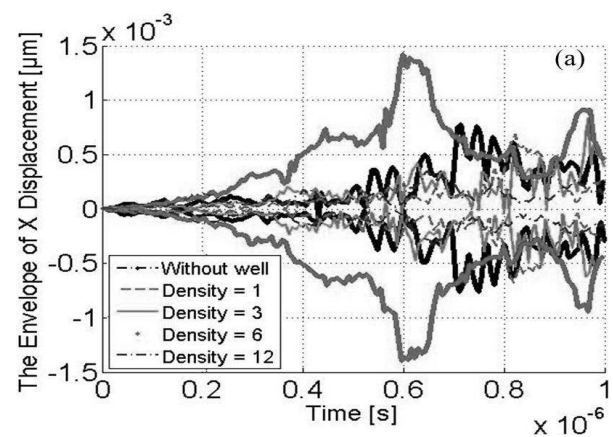

(a) Shear vertical wave

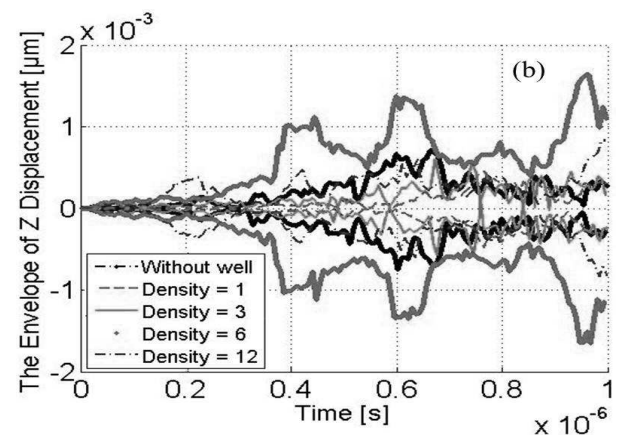

(b) Longitudinal wave

Fig. 5. Cross-sectional envelops of displacement profiles for the Rayleigh mode with different fluid densities.

The contour plot for envelops of total particle displacements at different liquid densities is shown in Fig. 6a. The mechanical wave at density $6 \mathrm{~g} / \mathrm{cm}^{3}$ is more strongly excited. Nevertheless, in Rayleigh mode, amplitude ratio at density $3 \mathrm{~g} / \mathrm{cm}^{3}$ reaches a peak (in Tab. 2). When the mechanical wave is converted into the output electric signal; output voltage is thus influenced. When electrical potential of density $6 \mathrm{~g} / \mathrm{cm}^{3}$ reaches the highest value, the potential difference between consecutive IDTs is lower than output voltage of device without well. Fig. 7 also shows that power spectral density of SAW structure without well roses to a peak.

Also, Fig. 8 shows the amplitude ratio and phase shift of surface acoustic wave of the sensing channel with different liquids. It drives to changes in the electrical output such as amplitude and phase. For the mechanical wave and electrical signal, the lowest peak of amplitude ratio is achieved at density $6 \mathrm{~g} / \mathrm{cm}^{3}$. However, Fig. 8 and 9 show that while phase at $12 \mathrm{~g} / \mathrm{cm}^{3}$ is always faster, phase at $6 \mathrm{~g} / \mathrm{cm}^{3}$ moves slower than that of device without well. Phase of SAW is most affected by density at $3 \mathrm{~g} / \mathrm{cm}^{3}$. 


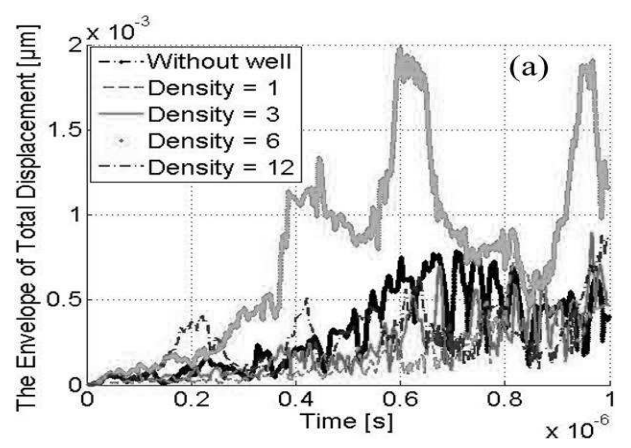

(a) Total displacement envelops of points placed behind the well for stationary fluids

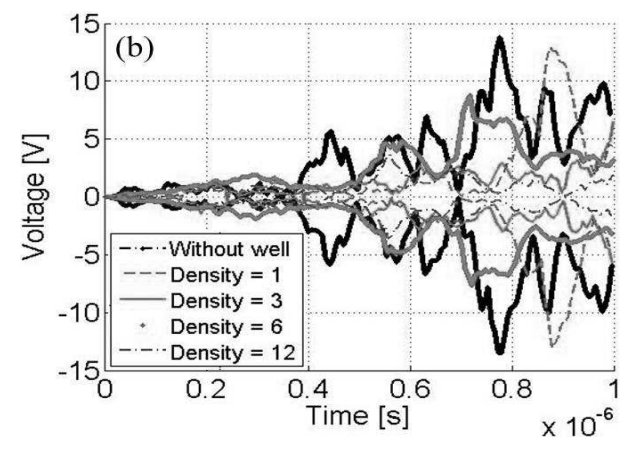

(b) Voltage envelops between 2 IDTs for standing fluids

Fig. 6. Mechanical and electrical properties of SAW.

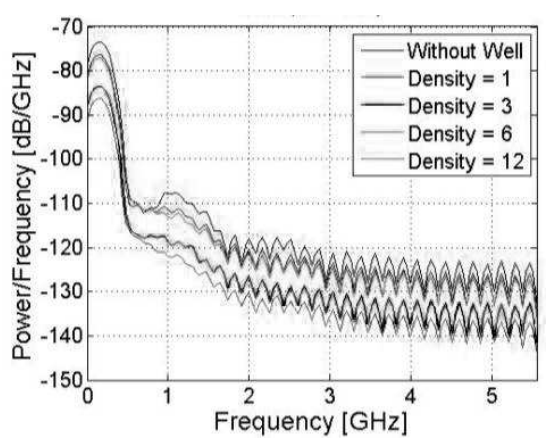

Fig. 7. Power spectral density (PSD) of output voltage.
Table 2. Amplitude ratio in Rayleigh mode

\begin{tabular}{|c|c|c|}
\hline $\begin{array}{c}\text { Density } \\
\left(\mathrm{g} / \mathrm{cm}^{3}\right)\end{array}$ & $\begin{array}{c}\text { Max } \\
\left(\mathrm{W}_{3} / \mathrm{W}_{1}\right)\end{array}$ & $\begin{array}{c}\text { Average Value } \\
\left(\mathrm{W}_{3} / \mathrm{W}_{1}\right)\end{array}$ \\
\hline 0 & 1779.1 & 13.5948 \\
1 & 258.9147 & 10.5177 \\
3 & $3.3725 \mathrm{e} 7$ & $97.513 \mathrm{e} 3$ \\
6 & 2402.1 & 10.4176 \\
12 & 1022.8 & 10.9042 \\
\hline
\end{tabular}

From Fig. 10, the time delay is an increasing function when the investigation time is long. Nevertheless, the electrical attenuation at these density values does not comply with this rule. Liquids with density $3,12 \mathrm{~g} / \mathrm{cm}^{3}$ result in the highest insertion loss for the sensing channel (Fig. 11). The cause is the excessive attenuation of shear waves at the liquid media.

\subsection{Investigating Moving Group}

This group is simulated within 200 nsec. Flow plays a role in excitation impact when it travels across the well and thus dissipated power of Rayleigh wave is less. The more velocity presents, the less this mode generates compressional waves like Fig. 12 in the short time.

According to the stream existence along $X_{3}$-axis, the total displacement is excited as shown in Fig. 13. It results in a near linear increase in the amplitude ratio of SAW motion for both the mechanical and electrical field. It leads to phase shift. However, Fig. 14 shows that potential difference between alternating IDTs of runoff is smaller than that of the reference channel. 


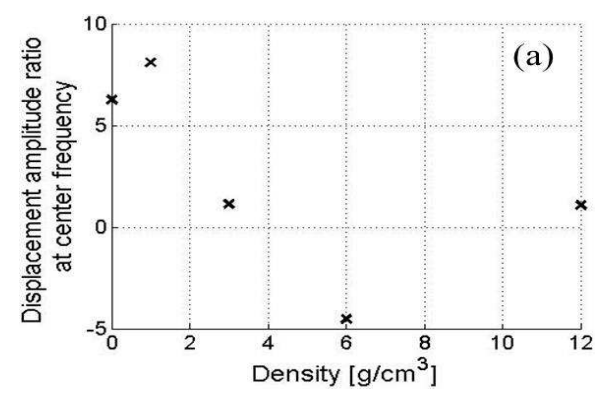

(a) Amplitude ratio

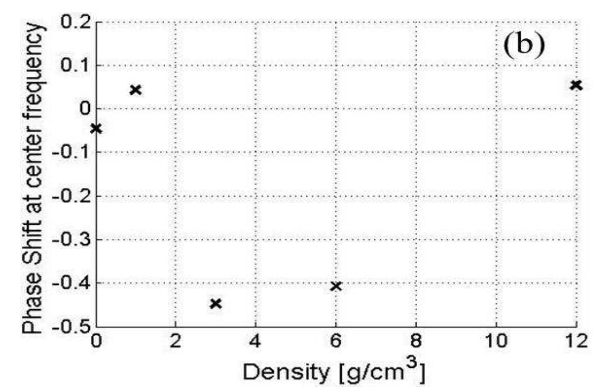

(b) Phase shift

Fig. 8. Displacement frequency response of the mechanical wave for standing liquids.

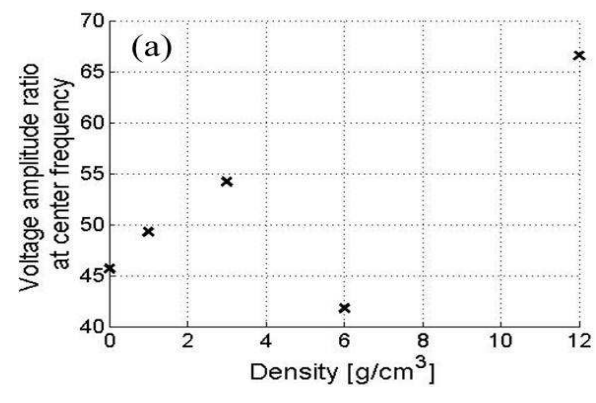

(a) Amplitude ratio

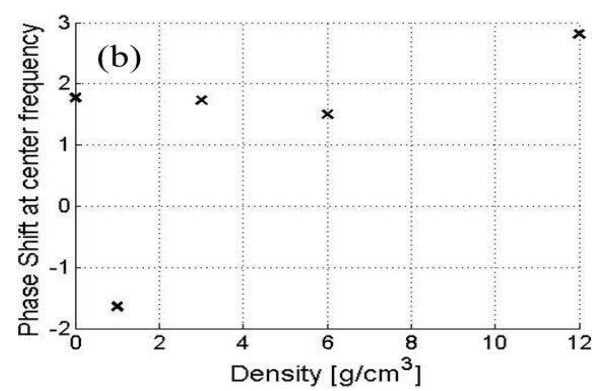

(b) Phase shift

Fig. 9. Voltage frequency response of the electrical signal for standing liquids at center frequency.

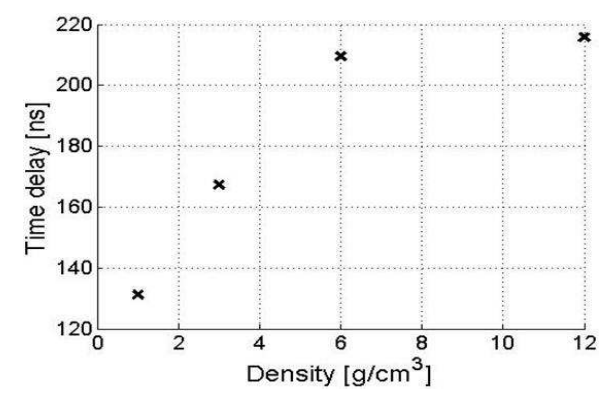

Fig. 10. Compared to system without well, time delay of system with well having liquid with density $1,3,6$, and $12 \mathrm{~g} / \mathrm{cm}^{3}$.

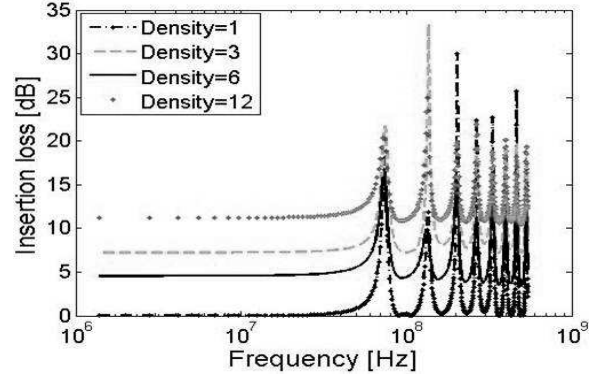

Fig. 11. Electrical attenuation response (shown as insertion loss) for the SAW device with liquid density $1,3,6,12 \mathrm{~g} / \mathrm{cm}^{3}$. 


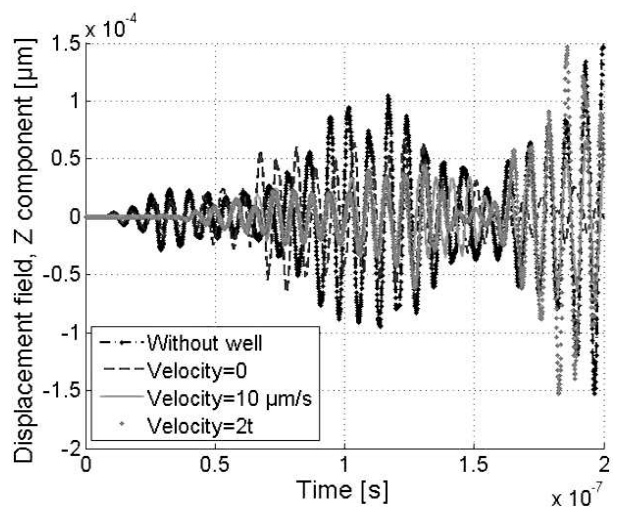

Fig. 12. Cross-sectional displacement profiles for the compressional wave mode with moving fluid within 200 nsec.

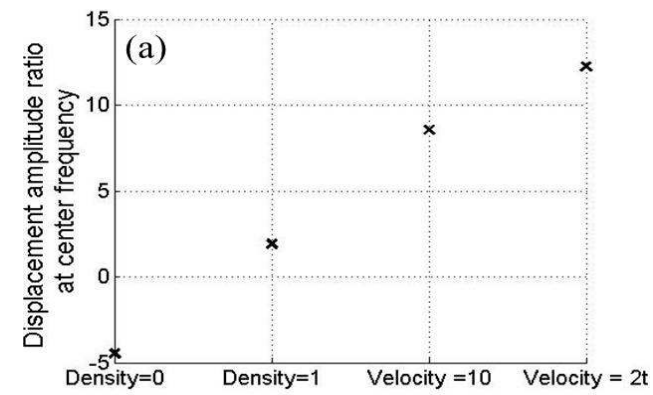

(a) Amplitude ratio

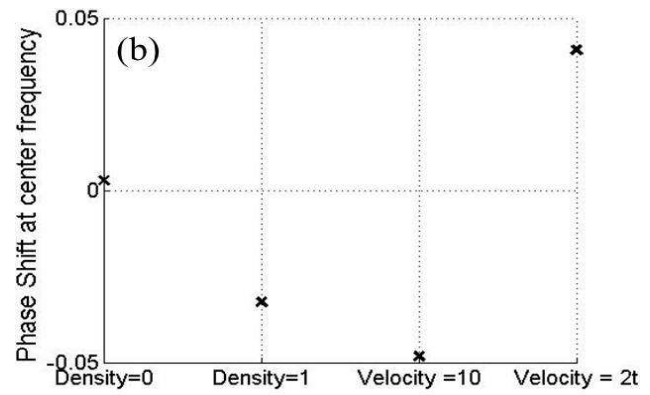

(b) Phase shift

Fig. 13. Displacement frequency response of the mechanical wave for the moving group at center frequency.

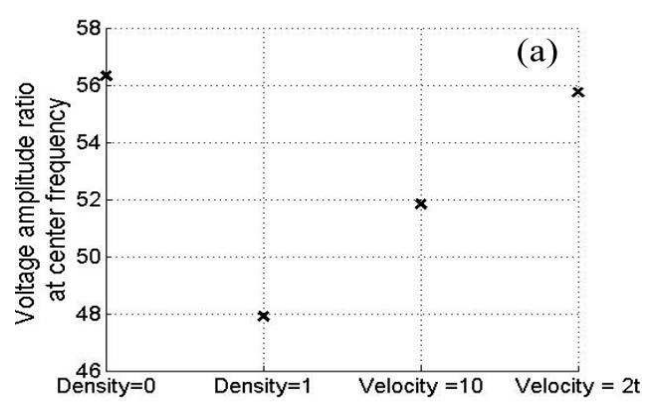

(c) Amplitude ratio

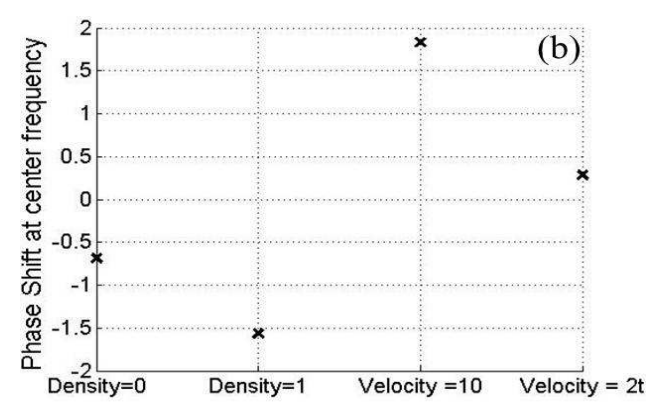

(d) Phase shift

Fig. 14. Voltage frequency response of the electrical signal for the moving group at center frequency. 
When the time delay of the stationary liquid is compared without well, it is much larger than that of the moving liquids with the same density as above Fig. 15. The flow affects the shear wave component and thus insertion loss of the moving liquid is higher than that of the standing liquid as shown in Fig. 16. When velocity is increased, the insertion loss is also moved up. Hence, besides density, runoff plays a role in influencing amplitude ratio, phase shift, time delay and attenuation of the surface acoustic waves and electrical output signal.

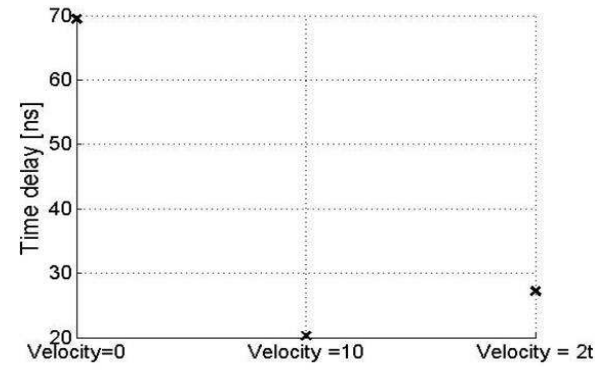

Fig. 15. Compared to system without well, time delay of system with well having velocity $=0,10,2 \mathrm{t} \mu \mathrm{m} / \mathrm{s}$.

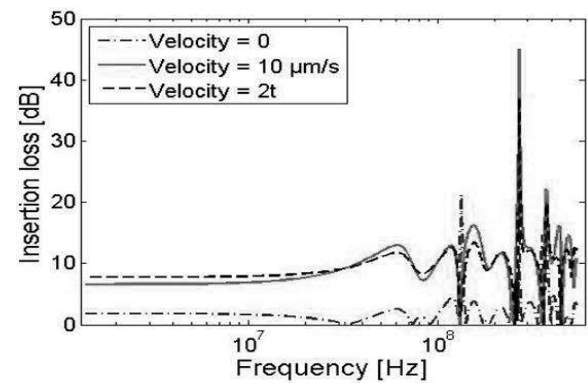

Fig. 16. Attenuation response (shown as insertion loss) for the single-crystal AlN substrate when changing velocity.

However, a fine mesh is required for accurate modeling of the SAW liquid sensor. It is necessary to increase the number of investigation cases for density and velocity. Also, another problem is the simulation time. It will increase with mesh size. Acoustic waves reflect from edges if the simulation time is sufficiently long. Although long simulation time is necessary to attain a stable state, it results in wave reflections causing instabilities. So, simulation time of $1 \mu \mathrm{s}$ and $200 \mathrm{nsec}$ is the good way to optimum it.

\section{CONCLUSIONS}

This paper presents a sensing system using a Rayleigh wave mode for the stationary liquid and moving group. They are highly influenced by the liquid density and achieve attenuation peak at density $3 \mathrm{~g} / \mathrm{cm}^{3}$ but in the $X_{3}$ axial displacement it is very low. However, the mechanical wave is excited most at density $6 \mathrm{~g} / \mathrm{cm}^{3}$. The 3D finite element analysis is performed to investigate the performance of the R-SAW sensor for liquid within longer simulation time. The response of SAW devices under alternating-current excitation proved that it is very sensitivity with small changes of the liquid if the well is placed in the middle of the two-port SAW delay-line device. This study provides a strong basis for manufacturing and designing $\mathrm{R}-\mathrm{SAW}$ sensors in practice.

\section{ACKNOWLEDGEMENT}

The authors would like to acknowledge the support of Dr. Phung Chi Dung of the University of Engineering and Technology, Vietnam National University, Hanoi and Dr. Catalin Rusu of the Frankfurt Institute for Advanced Studies. 
This work is partly supported by National Foundation for Science \& Technology Development (Nafosted) project 103.99-2012.24.

\section{REFERENCES}

[1] D. S. Ballantine, R. M. White, S. J. Martin, A. J. Ricco and E. T. Zellers, G. C. Frye and H. Wohltjen, Acoustic wave sensors, Academic Press, (1997).

[2] D. Morgan, Surface Acoustic Wave Filters, Elsevier, New York, (1985).

[3] J. Zhu, Tunable Zinc Oxide Surface Acoustic Wave Devices Based on Acoustoelectric interaction, UMI dissertation publishing, (2008).

[4] C. Ying, Piezoelectricity in zinc oxide-based multilayer structures for sensor applications, New Brunswisk, New Jersey, Doctoral thesis 2008.

[5] A. N. Cleland, Foundations of Nanomechanics from Solid-State Theory to Device Applications, Springer, (2003).

[6] B. D. Tung, B. Thu-Hang, N. T. Dat and C. D. Trinh, R-SAW Analysis on Single-Crystal AlN Substrate for Liquid Sensors, International Conference on Engineering Mechanics and Automation, (2012).

[7] S. Shiokawa and J. Kondoh, Surface acoustic wave sensors, Jpn. J. App. Phys., Part 1, (2004), 43, 2799-2802.

[8] S. Shiokawa, Y. Matsui and T. Ueda, Liquid streaming and droplet formation caused by leaky Rayleigh waves, IEEE Ultrasonics Symposium, (1989), pp. 643.

[9] B. Thu-Hang, B. D. Tung, N. T. Dat and C. D. Trinh, 3-D Finite Element Modeling of SAW sensing system for liquids, 2012 IEEE/ASME International Conference on Advanced Intelligent Mechatronics, p.782-787.

[10] A.N. Cleland, Foundations of Nanomechanics. From Solid-State Theory to Device Applications, Springer 2002, ISBN 3540436618.

[11] G. Chung, D. T. Phan, Finite Element Modeling of Surface Acoustic Waves in Piezoelectric Thin Films, J. of Korean Phys. Society, 3(57), (2010).

[12] G. Bu, D. Ciplys and M. S. Shur, L. J. Schowalter and S. B. Schujman, Leaky Surface Acoustic Waves in single-Crystal AlN Substrate, International J. of High Speed Electronics and Sys., 3(14), (2004), 837-846.

[13] A. Takayanagi, K. Yamanouchi and K. Shibayama, Piezoelectric leaky surface wave in $\mathrm{LiNbO}_{3}$, Appl. Phys. Lett., 5(17), (1970), 225-227.

[14] K. Yamanouchi and M. Takeuchi, Application for piezoelectric leaky surface waves, Ultrasonics Symposium, (1990), pp. 11-18.

[15] S. Tonami, A. Nishikata and Y. Shimizu, Characteristics of leaky surface acoustic waves propagating on $\mathrm{LiNbO}_{3}$ and $\mathrm{LiTaO}_{3}$ substrates, Jpn. J. Appl. Phys., Part 1, 5B(34), (1995), 26642667.

[16] A. Leon-Garcia, Probability and Random Processes for Electrical Engineering, Addison-Wesley Publishing Company, 2nd Ed.

[17] Z. Gajic, Linear Dynamic Systems and Signals, Prentice Hall, (2003).

[18] J. G. Proakis and D. G. Manolakis, Digital signal processing Principles, Algorithm and Applications, Prentice Hall, (1996).

[19] J. G. Gualtiei, J. A. Kosinski and A. Ballato, Piezoelectric materials for acoustic wave applications, IEEE Tran. on Ultrasonics, Ferroelectrics and Frequency control, 1(41), (1994).

Received July 31, 2012 


\section{APPENDIX}

Material constants for a non-conducting, non-viscous liquid and Aluminium Nitride piezoelectric in class $6 \mathrm{~mm}$ symmetry in simulation [19]:

$$
c^{f}=\left[\begin{array}{llllll}
c^{f} & c^{f} & c^{f} & 0 & 0 & 0 \\
c^{f} & c^{f} & c^{f} & 0 & 0 & 0 \\
c^{f} & c^{f} & c^{f} & 0 & 0 & 0 \\
0 & 0 & 0 & 0 & 0 & 0 \\
0 & 0 & 0 & 0 & 0 & 0 \\
0 & 0 & 0 & 0 & 0 & 0
\end{array}\right]
$$

$\rho^{f}=1000 \mathrm{Kgm}^{-3}, c^{f}=2.25 \mathrm{GPa}$ and $\nu=1480 \mathrm{~ms}^{-1}$. 